\title{
A Web Based E-Learning and E-Psychology Modular Environment
}

\author{
Athanasios S. Drigas, Leyteris G. Koukianakis and Yannis V. Papagerasimou
}

\begin{abstract}
This paper presents an electronic environment which supports activities of e-learning, as well as activities of epsychology. This e-environment supports synchronous and asynchronous activities of teaching and learning through communicative and informative tools and services. These "tools and services" are constructed in a powerful modular environment which can be adjusted and modulated to support different scenarios - policies of teaching, learning and manipulating the knowledge circle, in an electronic way. On the other hand, the ideas of learning, of transferring the knowledge, of analyzing the end user (learner) needs, of analyzing the status and end user development, on supporting synchronous and asynchronous communication, between the learner and teacher professor parts, are also included in the e-psychology circle and are procedures of it. As a result the e-learning and the epsychological actions, like psychological support and consultation, are parallelized, and can be both supported by the aforementioned e-environment.
\end{abstract}

Index Terms-e-learning, e-psychology.

\section{INTRODUCTION}

$\mathrm{T}$ HE Internet and the World Wide Web are changing the very nature of our society in ways unparalleled since the industrial revolution. This is affecting local, national and global economies and their infrastructures. Information and learning content is available at any time, at any place, and to any Internet user. This is creating a tremendous opportunity for academic institutions and industrial training organizations to provide on-demand web based education and training through course delivery platforms such as the Ariadne Web based learning environment and electronic textbooks through the use of authoring tools such as InterBook [1,2]. Despite this, e-learning and e-psychology cannot fully replace the traditional learning and counseling processes. As a result, a new scientific term was introduced namely, hybrid learning and hybrid psychology. The scientific term hybrid learning

Manuscript received December 14, 2006.

A. S. Drigas is the Scientific Coordinator of Net Media Lab of N.C.S.R. 'Demokritos', Agia Paraskevi, 15310, Athens, Greece (Phone: +302106503124; Fax: +302106532910; e-mail: dr@imm.demokritos.gr).

L. G. Koukianakis is the Head of Network and Multimedia Applications of Net Media Lab of N.C.S.R. 'Demokritos', Agia Paraskevi, 15310, Athens, Greece (e-mail: kouk@imm.demokritos.gr).

Y. V. Papagerasimou is an Electronic and Computer Engineer of Net Media Lab of N.C.S.R. 'Demokritos', Agia Paraskevi, 15310, Athens, Greece (e-mail: ypapa@imm.demokritos.gr). suggests the integration of e-learning with traditional learning processes, while similarly the term hybrid psychology suggests the integration of e-psychology with traditional psychology processes.

Psychology, as well as other disciplines, is not unaware of the rapid advances of the Informatics Science as well as its positioning as a vital tool for study and development of all knowledge and science fields [3,4]. Hence, psychology makes use of this tool to create programs for psychological intervention, as a means of prevention [5,6,7,8,9], assessment $[10,11]$, orientation, and specialized counseling. The most widely used means were e-mail, web pages and chat rooms. At the same time, these information tools are used for learning by offering teleconferences, forums, virtual classrooms, and other channels [12,13].

There is concern about the quality of the information obtained from websites, as anyone can publish information on the Internet $[14,15,16,17]$. However, in one study that sampled actual patients as compared to researchers conducting web searches, supported persons used many different websites for health information and consistently rated as their favorite websites those recognized to be reputable (i.e., from prominent universities, government organizations, or other recognized prominent health organizations) [3]. Also, the health information on the Internet has a positive psychological impact on those who use it, as the use of the Internet for health information among supported persons was found to be associated with greater social support and less loneliness $[18,19]$. All this suggests the need for clinicians to recognize this new medium and incorporate it into their assessment and treatment methods.

In addressing the above context, we developed a course delivery platform for e-psychology that is based on the active utilization of:

- the principles of the learner-centered paradigm of education and training that lead to the highest possible learner convenience in learning

- the principle of modularity of learning content (based on the reusable learning objects concept) that leads to great flexibility in composing different customized versions of an online course for various groups of trainees with different educational backgrounds, learning objectives, technical skills and past experiences

- an innovative Web-Based Instructional (WBI) tool 
that uses web-based streaming multimedia and various communication technologies and offers both online and offline modes of learning content delivery.

\section{ABSTRACT LEVEL DESCRIPTION}

Psychology uses learning and educative techniques in all the branches that it comprises (behavioral, psychodynamic, cognitive etc). These learning and educative procedures are used in various levels of the production and support of the psychological procedure. Thus, learning and education are used for the supported person level at the intervention procedure as well as at the level of organizing the psychology cycle and finally, at the level of the constant upgrade of the psychologists' and counselors' skills.

On the other hand, the e-learning procedure has proven that it can successfully use the ICTs for the supporting of learning, education and training in all the sectors and levels of the educational system as well as of lifelong learning and training.

The successful e-learning techniques are embedded in the system and it is proven that they can be used in the same successful way for the improvement of the psychology services provision cycle (to the end-user). The e-learning techniques used in the psychology services provision cycle are used in two axes.

Firstly, to support learning and education, which are indispensable in all the branches of psychology and secondly, to change entirely the form of the provision of the psychological cycle services to the end-user, since the tools that are used for e-learning (in a new form and role now) are used to support the operations, procedures and services of the psychological cycle.

Thus, a complete e-psychology environment can offer and support a large sum of the operations and services of the psychological cycle such as tests, evaluation, diagnosis, support and intervention among the supported persons and the psychologists while simultaneously, it offers all the necessary learning and educational procedures to this heterogeneous community of recipients (psychologists, counselors and supported persons).

Such an e-psychology system is usually used to supplement and improve parts of the psychology services provision cycle without substituting the personal contact between the psychologist and the supported person. For this reason, this kind of systems are called hybrid e-psychology systems. In other words, this can be presented as the psychology cycle that is provided through the integration of traditional procedures with the new electronic ones.

The aim of the platform is the integration of the ICTs with the traditional learning and psychology processes for the development of a hybrid system that supports hybrid learning as well as hybrid psychology (Fig. 1).

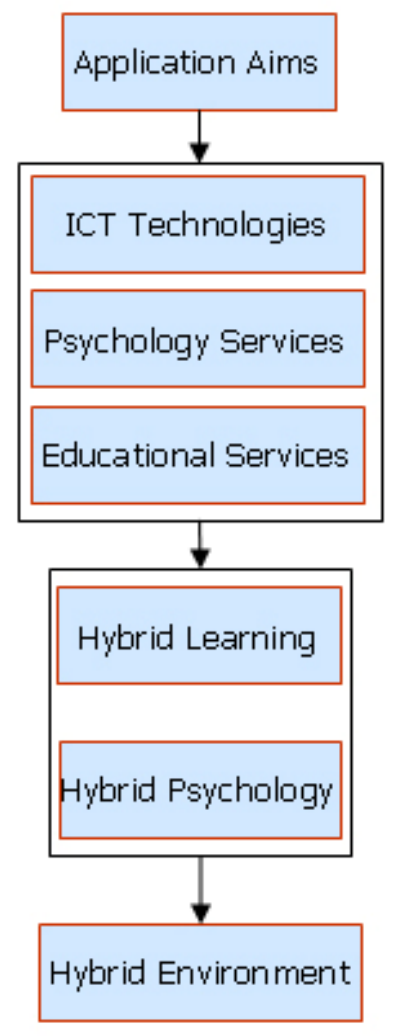

Fig. 1. Logical structure

As far as the ICTs are concerned they are distinguished into two major service categories. The "Informative" and "Communicative" services. The latter are divided into two services subcategories. The synchronous and asynchronous services, which determine the terms "synchronous" and "asynchronous" environments (Fig. 2). These technologies were used for the development of the hybrid system imprinting the traditional learning and psychology processes with "synchronous" and "asynchronous" learning and counseling tools in the system.

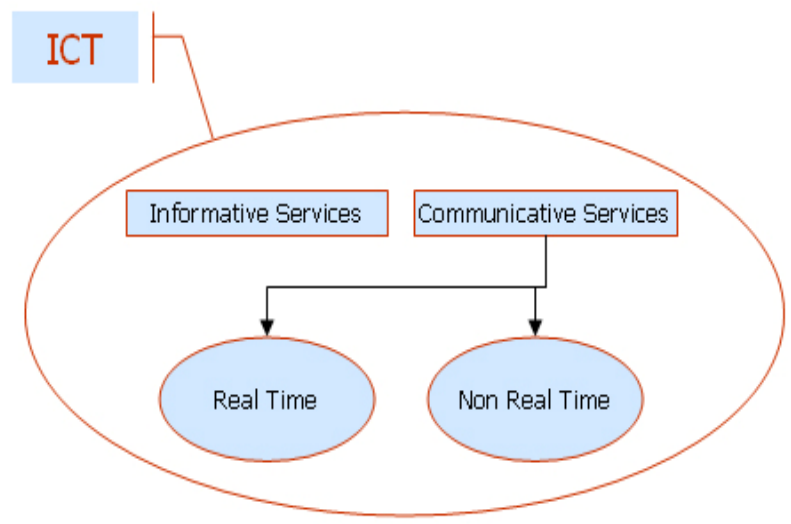

Fig. 2. ICT services

Psychology is distinguished into six major branches with respect to the cause of ones unusual behavior. All psychology branches refer to specific target groups based on unusual behavior problems. The method that each branch uses differs 
from the others while the processes (evaluation tests, diagnosis and therapy) are the same in all branches (Fig. 3).

The possibility of supporting personal teaching - learning as well as supporting classes or larger entities like levels of learning or virtual schools finds also similarities and proportions to the hybrid psychological circle, with the possibility of supporting psychological consultancy in a personal way or in a small group, or larger handling entities of them, like therapy directions, or virtual psychological health centers.

The result of the ICT integration with the psychology processes is depicted in fig. 4. This integration of the psychology branches in a web-based environment is available to the Internet user. The methods and processes that each branch follows are materialized through electronic tools such as: discussion forums, message boxes, video and audio conference etc. Similarly, the learning methods and processes are introduced into the same web-based environment through informatics tools.

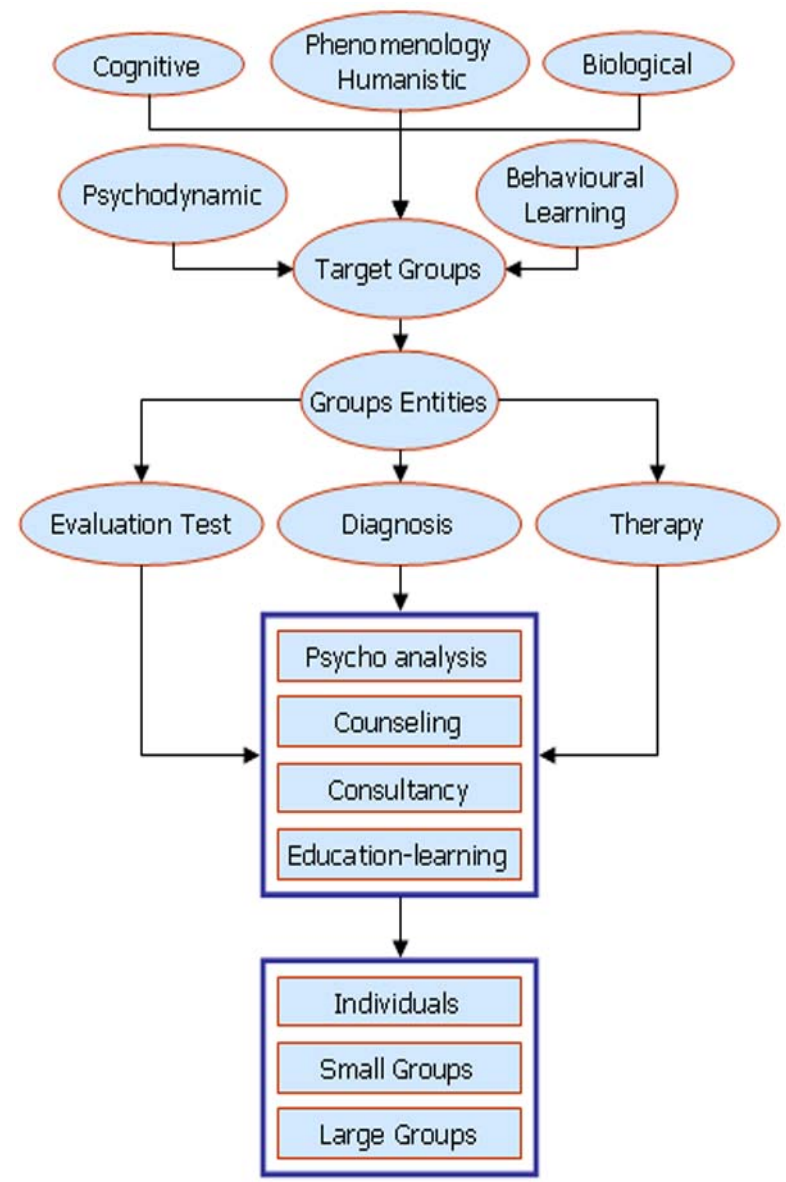

Fig. 3. Psychology services

Moreover, the user levels and user interfaces of the electronic environment are parallelized as follows: the elearning and hybrid learning administrator, instructor, student and user, support similar actions with the e-psychology and hybrid psychology administrator, psychologist, supported person and user.
Finally, the seminars, classes, courses and educational material from the hybrid learning circle, are supported by the same tools in similar procedures with the therapeutic entities, small groups, therapeutic process, and supporting material, from the hybrid psychology circle.

The specifications of the user levels, the e-content and the e-tools in an e-psychology and hybrid psychology platform can be easily implemented through a simple correspondence of the psychology ontologies to the generic e-learning and hybrid learning ontologies.

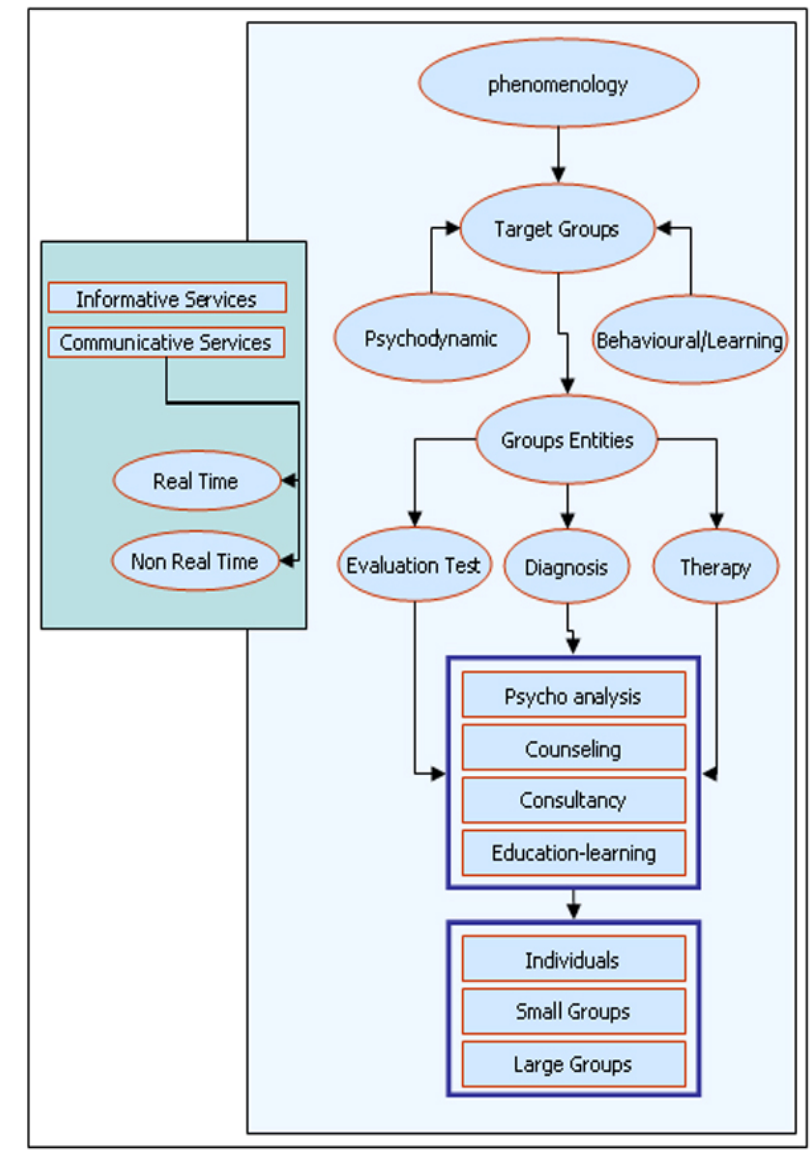

Fig. 4. Hybrid psychology structure

The way that the e-learning and hybrid learning tools are related to the correspondent e-psychology and hybrid psychology tools, is presented in fig. 5. From fig. 4 above, it is obvious that the roles of the "instructor" and the "student" are transformed into the roles of the "psychologist" and the "supported person" respectively. The "classes" are turned into "small therapy groups" and the "courses" into "supporting material”, The "consulting material” (examples, exercises, multiple choice tests) correspond respectively to diagnosis, educational exercise for treatment and diagnostic tests. Finally, all the tools (web directory, glossary, references, video and audio lectures, events calendar, news, announcements, mailing lists, e-library, message box, e-mail, video and audio e-mail, video and audio conference, discussion forums, instant messaging, chat, and telephony) can be easily applied in both platforms. 


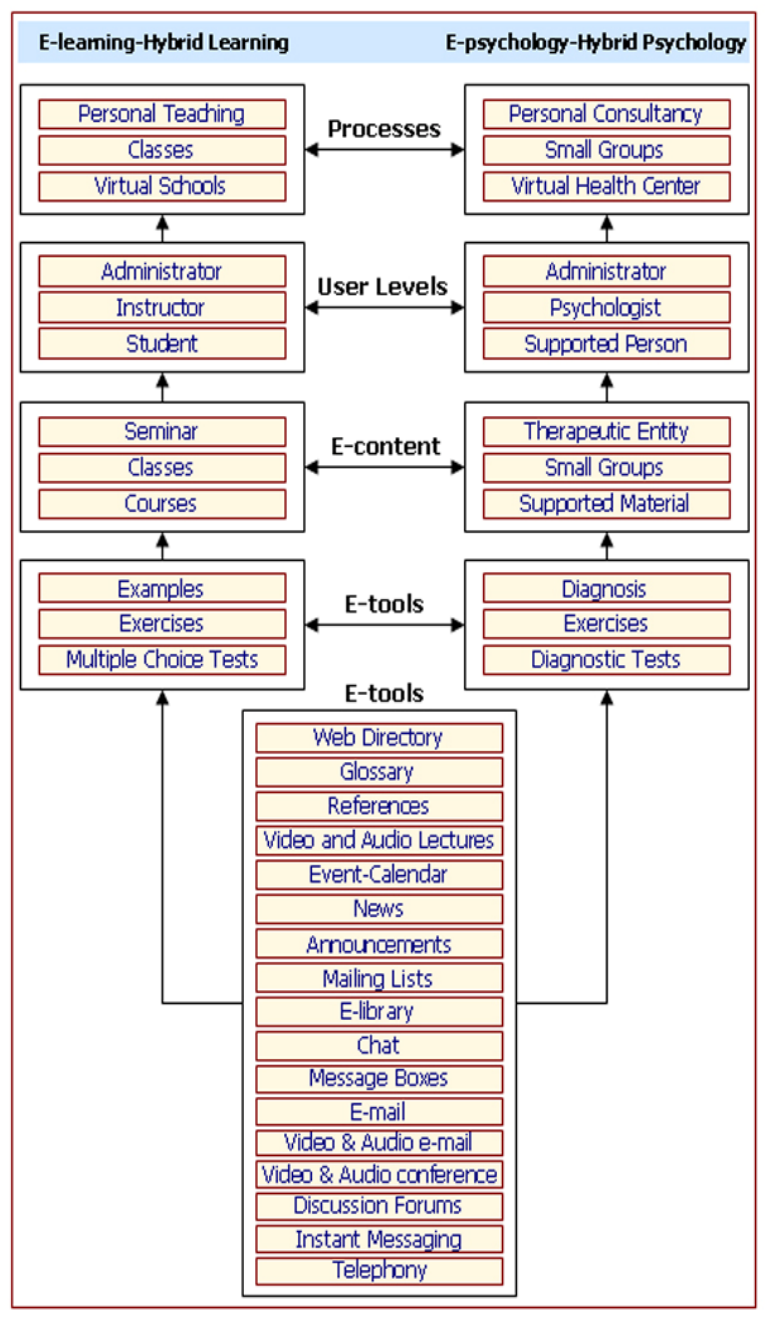

Fig. 5. Modular environment

\section{SYSTEM ANALYSIS}

\section{A. Environment Tools}

The environment includes tools that offer flexibility and adaptability depending on their use. The design of these tools was based on existing web services, such as discussion forums, chat, message box, e-libraries, which are widespread in the public web community. These tools are distinguished into two major groups: “Informative"' and " Communicative"'. The former are divided further into two subcategories: the "Informative" and the "Supportive" tools, which include services related to the educational-supporting material and its presentation. Similarly, the latter, are also divided into two subcategories: "Synchronous" and "Asynchronous" tools, which include services that allow the communication between different user groups (users belonging to a different session level).

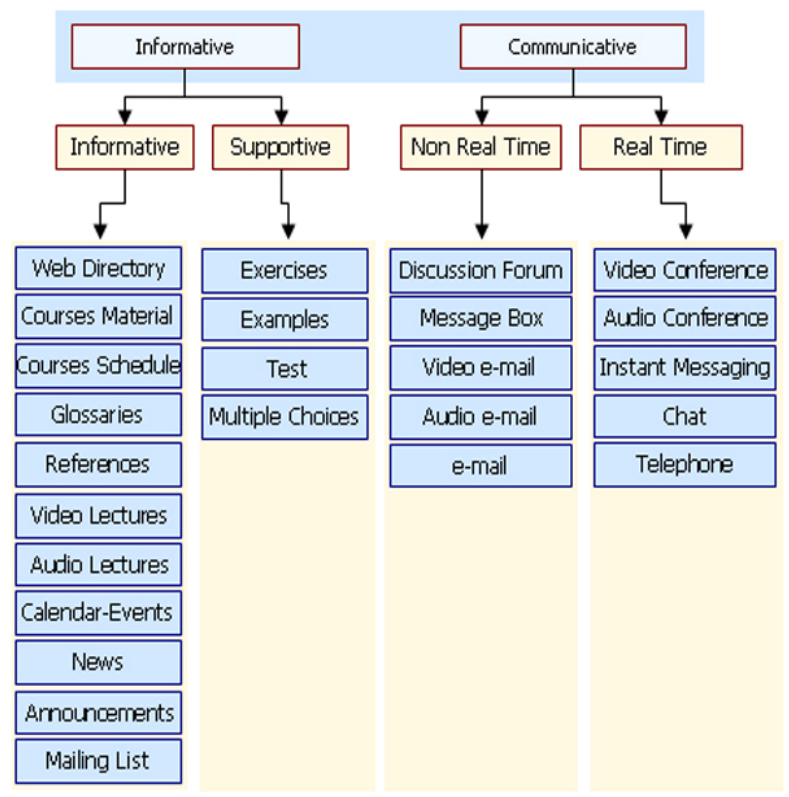

Fig. 6. Informative and communicative tools

The environment offers the possibility of management of these tools according to the user groups' permission. More explicitly, the "Informative" tools are the following: web directory, courses material, courses schedule, glossaries, references, video and audio lectures, calendar-events, news, announcements, mailing lists. On the other hand, the "'Supportive'” tools are: exercises, examples, Multiple Choice Tests. As far as the "Communicative” tools are concerned, in the "synchronous" subcategory the tools are: video and audio conference, instant messaging, chat, telephony. One the other hand the "asynchronous" tools are: discussion forums, message boxes, e-mail, video and audio e-mail. The aforementioned information is depicted in fig. 6 and relates to both hybrid learning and hybrid psychology. Finally, it must be noted that the environment relates the tools to the educational material courses according to the specific user level permissions. These levels are analyzed in the following sections.

\section{B. User Levels}

Four user levels are distinguished in the environment, each of which uses different supporting tools. Depending on the corresponding use, these levels have a different role: Administrator, Instructor-Psychologist, Student/Supported Person and Visitor. The user levels for hybrid learning and elearning correspond to the relative user levels of hybrid psychology and e-psychology. That is to say, that every administrator whether for e-learning or for e-psychology has the same tools and services at his/her disposal as well as the same restrictions. Each of them interacts with the other through the "informative", and "communicative" tools related to each level.

\section{Administrator}

The administrators coordinate and manage the corresponding application through the corresponding 
administrative tools, depending on whether they are e-learning or e-psychology administrators. They also determine which user level-group has the permission to use the corresponding "Informative" and "Communicative" tools. Moreover, the administrator can communicate with the instructor or psychologist in order to be kept informed about the progress of the Instructors'-Psychologists' courses and sessions.

\section{Instructor/Psychologist}

In this level, the instructors determine the educational and consulting material depending again on whether they are elearning or e-psychology instructors. In addition, they also determine the development and the way that the material will be presented to the students/supported persons and to the entire Internet community. The instructors are able to produce the e-content of their course, aiming at the better comprehension and assimilation of it from their students/supported persons as well as the visitors. Finally, the communication between the instructors and the students/supported persons aims at the resolution of questions that rise during the courses and sessions.

\section{E. Student/Supported Person}

The students/supported persons determine the successful development of the seminars and courses. Moreover, they read the educational-psychological material registered by the instructor, and communicate and pose questions to the instructor who is responsible for resolving the exercises and multiple choice questions. A remarkable fact is that the students/supported persons have access to all the information and services, regardless of when they undertook a certain course-session, which results in the better development and understanding of the courses-sessions.

\section{F. Visitor}

In this level, the visitors can easily browse the e-content of the corresponding courses-sessions. The innovation of this level is the fact that the visitors can have access not only to the consulting material for each course-session, but also to the discussion forums between the instructors and the students/supported persons (carried out during courses or during practical applications and which include general and not confidential discussions). Finally, this level follows the philosophy-standard "knowledge-access for all" giving the permission to the random visitors to obtain the knowledge of their object of interest. The visitors can have access to non confidential information only, which is available for educational purposes. Confidential information is protected and can only be accessed by authorized people in order to respect and protect personal information.

\section{Pilot Project}

The presented environment was developed under the framework of the Greek research program, namely, "Conditions Improvement of Inclusion to Educational System of Individual with Multiple Handicaps"' that was funded by the O.P.E.I.P.T. European Community Program (Operational Program of Education and Initial Professional Training) [20]. The projects' main objective was the training of special education teachers on multiple handicaps issues. The work included two seminar periods: "training" and "specialization". The former included training in physical classrooms while the latter included training in physical classrooms as well as e-learning. This hybrid environment was parameterized and configured properly, to conform to the project specifications and needs. In this way, it assured the distance training form and also supported the dissemination of new ideas and knowledge in the special education community.

During the two seminar periods two questionnaires were handed to the participants, which comprised of one word answers (yes/no/other) mostly. The first questionnaire was handed before the pilot implementation (training seminar) of the project and concentrated on more general information regarding the participants' knowledge of computers, the Internet, the e-services as well as some general questions. On the other hand, the second questionnaire was handed to the participants after the pilot implementation (specialization seminar) of the project and the questions in it concentrated on the hybrid system and e-psychology.

The questionnaires were filled out by a total of 480 participants, 170 of which were male and 310 were female, from five major cities of Greece (Athens, Thessaloniki, Ioannina, Kavala and Heraklion). Among them, there were 105 nursery school teachers, 231 primary school teachers and 144 secondary school teachers. It is important to note that 158 participants came from Athens, while 104, 91, 53 and 74 came from Thessaloniki, Ioannina, Kavala and Heraclion respectively. The reason for this is that every city represents different economies, different cultures and different geographical areas of Greece, which have an impact on the digital division and digital illiteracy among the citizens of these areas.

The reason why the first questionnaire was used, was to gain in-depth information of the participants' level of knowledge as far as the ICTs are concerned in order to able to accurately define the sample into experience groups. Similarly, the second questionnaire was used in order to fully explore the participants' viewpoint on the hybrid system, the future use of such a system in the psychology sector and the possible barriers and flaws that the users encountered and were forced to resolve. Both questionnaires are depicted in the following fig. 7. 


\section{Interview Questionnaire}

\begin{tabular}{|c|c|}
\hline Issue & Question \\
\hline General & $\begin{array}{l}\text { 1. Sex } \\
\text { 2. Education Level where you belong? }\end{array}$ \\
\hline Computer/Internet Skills & $\begin{array}{l}\text { 3. Do you know how to use a Computer (Windows, } \\
\text { MSoffice)? } \\
\text { 4. Do you know how to use the internet? } \\
\text { 5. Why do you use (or will you use in future) the internet? } \\
\text { 6. Why don't you have access to the internet? } \\
\text { 7. Would you like to have more "friendly" Information on } \\
\text { the Internet? }\end{array}$ \\
\hline$t$-services & $\begin{array}{l}\text { 8. Do you believe that the New Technologies will help you } \\
\text { in your job? } \\
\text { 9. Are you familiar with the e-learning and e-psychology } \\
\text { concepts? } \\
\text { 10. Are you interested in the future to learn one or all of the } \\
\text { above concepts? } \\
\text { 11. Do you believe that the e-learning, e-psychology and } \\
\text { other e-services would help you in your job? }\end{array}$ \\
\hline Pilot / Hybrid System & $\begin{array}{l}\text { 12. What made you participate in the pilot implementation } \\
\text { of the hybrid systems projedt? } \\
\text { 13. Do you believe that the hybrid system served your } \\
\text { needs? } \\
\text { 14. How did the hybrid system serve your needs? } \\
\text { 15. Do you believe that the hybrid learning system offers } \\
\text { equal capabilities than the traditional classroom? } \\
\text { 16. Do you believe that the hybrid system tools are } \\
\text { adequate? } \\
\text { 17. Do you believe that the quality of courses correspond to } \\
\text { your needs? } \\
\text { 18. How easy to use (easy navigation) and user friendly was } \\
\text { the hybrid system for you? } \\
\text { 19. Do you believe that the hybrid system helped towards } \\
\text { the upgrade of your computer and internet skills? }\end{array}$ \\
\hline E-psychology & $\begin{array}{l}\text { 20. Do you believe that the new technologies can penetrate } \\
\text { in the psychology sector? } \\
\text { 21. Do you believe that e-psychology can substitute } \\
\text { adequately (up to a point) the traditional relation } \\
\text { between psychologists-supported persons? } \\
\text { 22. Do you believe that the new technologies can penetrate } \\
\text { in the special needs schools? } \\
\text { 23. Do you believe that the anorymity that this e- } \\
\text { psychology system offers renders it more approachable } \\
\text { than the traditional psychological session? } \\
\text { 24. Do you find such systems secure and trustworthy } \approx \text { far } \\
\text { as the quality of the psychologists' answers is concerned } \\
\text { within the framework of e-psychology support? }\end{array}$ \\
\hline
\end{tabular}

Fig. 7. The two questionnaires

\section{A. Training Seminar Questionnaire}

As far as the first questionnaire is concerned, the following results were deduced. Firstly, the largest percentage of the interrogated participants $(37,5 \%)$ answered that they know how to use a computer well and $27 \%$ replied that they can use a computer very well, while only a $16.7 \%$ replied that they cannot use a computer at all. Almost the same percentages applied for the question 'Do you know how to use the Internet?'. It was interesting to notice that a $62,5 \%$ of the interviewed participants stated that the primary reason for not having access to the Internet was the very high cost., while only a $6,2 \%$ do not know what the Internet is.

Concerning the question 'Do you believe that the new technologies will help you in your job?', the largest percentage (68,75\%) answered positively, while a mere $10 \%$ replied negatively. A $73 \%$ of the participants stated that they are familiar with e-learning as a concept, but haven't used elearning in depth and only a $10 \%$ stated likewise for epsychology, while a $48 \%$ expressed their interest in the epsychology concept.

Finally, an extraordinary $62 \%$ of the interviewed participants believe that e-learning and e-psychology as well as other e-services could be of great help to them both in their professional and personal lives, while only $8,5 \%$ replied the contrary.

\section{B. Specialization Seminar Questionnaire}

As far as the second questionnaire is concerned, which had to do with the actual hybrid system and e-psychology on a more concrete basis, the following results were drawn. Firstly, concerning the question 'What made you participate in the pilot implementation of the hybrid system project', a $51 \%$ of the interrogated participants answered that the computers and Internet knowledge that they would acquire was the principle reason for joining in. The largest percentage (73\%) stated that the hybrid system served their needs and $41,5 \%$ served their needs due to the fact that they were able to access educational e-content regardless of their geographical area. As it can be seen in the following fig. 8, the participants who inhabit the smaller cities (Ioannina, Kavala and Heraclion) need these systems in order to have easily accessible e-content.

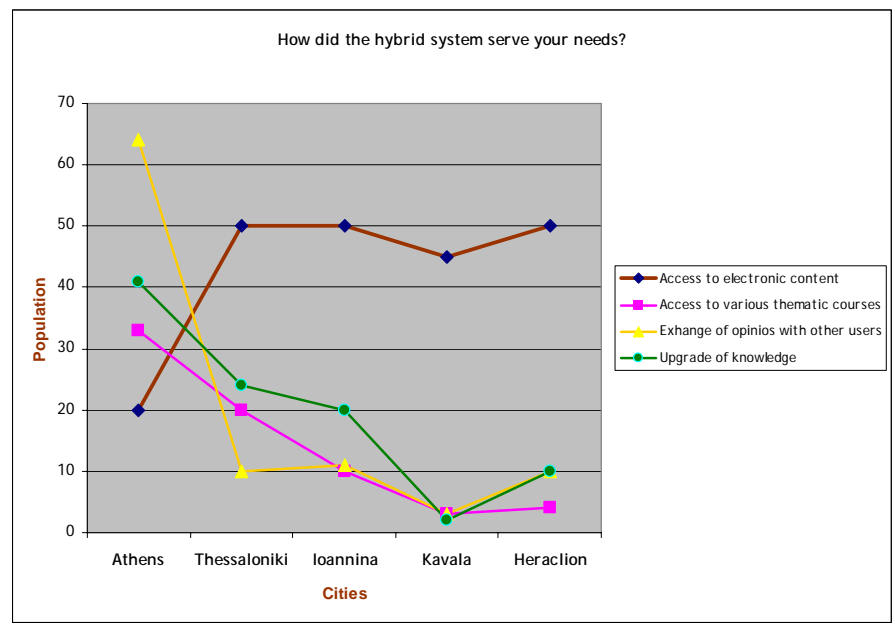

Fig. 8. How did the hybrid system serve your needs?

Moreover, a $42 \%$ replied that the hybrid learning system offers equal capabilities with the traditional classroom and $76 \%$ stated that the systems' tools were adequate enough, while a $72 \%$ find that the courses correspond to their needs. As far as the navigation and user-friendliness of the system is concerned, $60 \%$ found it easily navigable and user-friendly, while only $17 \%$ answered the opposite.

Furthermore, it was very important to deduce that $77 \%$ of the participants found that their participation in the pilot implementation of the hybrid system as well as the hybrid system itself helped them towards the upgrade of their computer and Internet skills, while only a mere $6 \%$ disagreed. Looking at fig. 9, one can easily observe that regardless of the city where the participants came from, they all agreed with the aforementioned comment. 


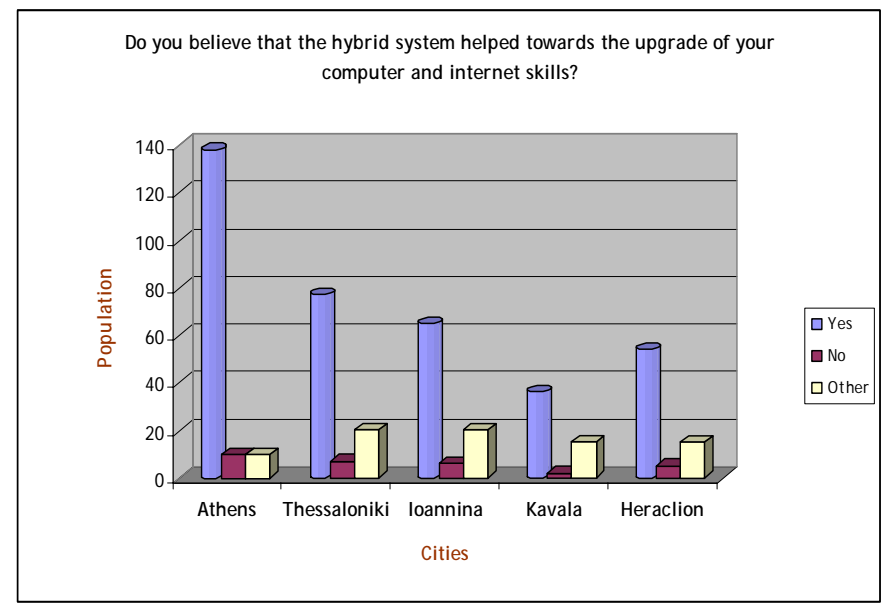

Fig. 9. Do you believe that the hybrid system helped towards the upgrade of your computer and Internet skills?

Finally, there were four questions regarding e-psychology, where the main observation had to do with the fact that the answers were quite balanced in relation with the previous questions about the hybrid system. In particular, what regards the question 'Do you believe that the new technologies can penetrate in the psychology sector', a $37,5 \%$ replied positively, while a $29 \%$ replied that it depends. Despite this, according to the $60 \%$ of the participants, it is an absolute necessity to introduce and penetrate the new technologies within the special needs schools where the teachers have a double role, that of a teacher and that of a psychologist.

On the other hand, a large percentage (49\%) believe that under specific circumstances e-psychology can substitute up to a point the traditional relation between psychologists and supported persons. The following fig. 10 proves that even in the capital (Athens), the people are doubtful whether epsychology has the potential to substitute the traditional psychology.

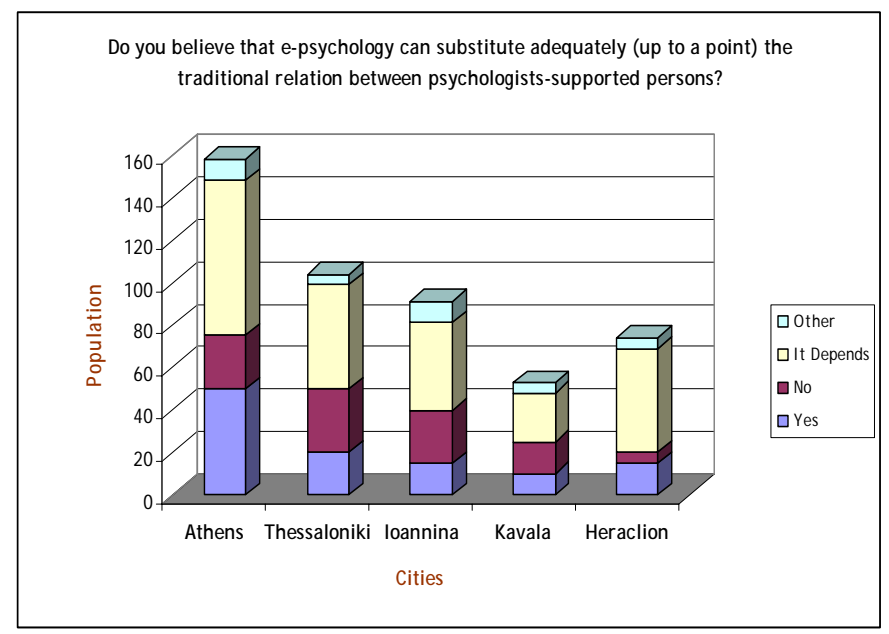

Fig. 10. Do you believe that e-psychology can substitute adequately (up to a point) the traditional relation between psychologists-supported persons?

The last two questions of the second questionnaire brought up some very interesting results. For a start, a 53,5\% believe that the anonymity that an e-psychology system can offer renders it more approachable than the traditional psychological session, while 46,5\% disagree. Fig. 11 depicts that the participants from the small cities (Ioannina, Kavala and Heraclion) where the societies are smaller and more traditional in their way of thinking, replied that the anonymity can make the e-psychology concept more approachable.

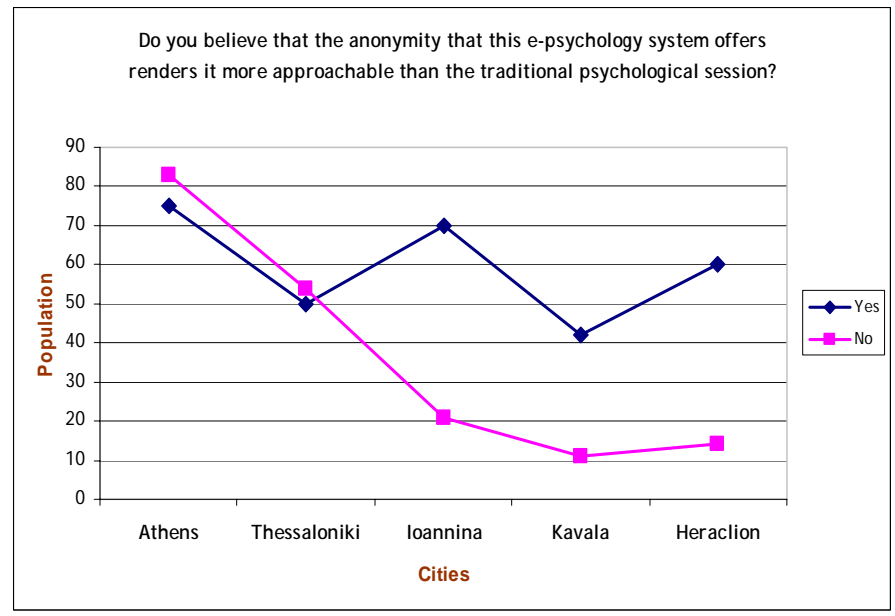

Fig. 11. Do you believe that the anonymity that this e-psychology system offers renders it more approachable than the traditional psychological session?

Lastly, when the participants were asked to state whether they found such systems secure and trustworthy as far as the quality of the psychologists' answers were concerned (within the framework of the e-psychology support), the answers were quite balanced. More particularly, a $44 \%$ answered positively, a $49 \%$ percentage negatively, while $7 \%$ did not answer at all.

\section{CONCLUSION}

The main advantage of the discussed platform is the fact that it offers the pioneering experience for either pair "teacher-student" or "psychologist-supported individual" to span two completely different worlds, the physical and online worlds, simultaneously. Undeniably, hybrid learning and hybrid psychology improve the traditional learning courses and psychology session's cycle. Moreover, what they specifically do is that they completely transform the cycle, as only a $10 \%$ of the traditional way of working is used, while the remaining $90 \%$ is all ICT based. The reason why $10 \%$ of the traditional cycle is kept lies in that it works as a feedback for the e-learning and e-psychology operator in order to make fine tuning and high quality adaptation of the procedure to the end user needs.

What makes hybrid learning - psychology worth while and innovative, is the fact that it gives the instructors (whether teachers or psychologists) the unique opportunity to experiment and incorporate e-services in their coursessessions. This has immediate results, in that it enables them to carry out their respective work traditionally, slowly incorporating technology while at the same time, it offers the opportunity to make their work more appealing and interesting 
for them as well as for their students/supported individuals. Undoubtedly, the use of ICT enhances the learningpsychology environment a great deal, making the learningpsychology process a whole new and beneficial experience for all.

This paper has made an effort to underline and pass the clear message that e-psychology is a new evolution of psychology and it is based on the techniques, tools and services of the well known e-learning procedures as it has been pointed out. Moreover, it is obvious that this paper underlines the possibility of e-psychology to be used for both technical applications as well as for didactic purposes.

The main contribution and innovation of this environment is that for the first time an organized e-psychology environment is presented in a methodical way, which exploits all of the informative and communicative abilities of the ICTs and proves that these procedures are identical and parallel to the corresponding procedures of e-learning. E-learning has successfully proven that it suits well the teaching and learning procedures. What is hoped for is that this e-psychology environment will be able to cover the needs of the psychologists as well as the supported persons in all the fields of psychology.

\section{REFERENCES}

[1] P. Brusilovski, J. Eklund, E. Schwarz, "Web-Based Education For All: A Tool For Development Adaptive Courseware”, Computer Networks and ISDN Systems, $7^{\text {th }}$ International WWW Conference, 14-18 April 1998, 30 (1-7), 291-300.

[2] R. V. Durm, E. Duval, B. Verhoeven, K. Cardinaels, H. Olivie, "Extending the Ariadne Web-Based Learning Environment", World Conference on Educational Multimedia, Hypermedia and Telecommunications (EDMEDIA) 2001, Norfolk, VA 2001

[3] V. García, L. Ahumada, J. Hinkelman, R.F. Muñoz, J. Quezada, "Psychology over the Internet: On-Line Experiences" CyberPsychology \& Behavior, Vol. 7 No. 1, pp. 29 - 33.

[4] R.R. Plant, A. Howes, N. Hammond, A. Trapp, "A Virtual Psychology Lab: A Look Back Over The Last Three Years", Computers in Psychology Conference 2000, University of York, UK, 29-31 March, 2000.

[5] I. Marks, S. Shaw, R. Parkin, "Computer-Aided Treatments of Mental Health Problems”, Clinical Psychology: Science and Practice, Vol 5, 1998, pp.151-170.

[6] M. Kenwright, "Computer-Aided Self-Treatment for Phobias", Computers in Psychology Conference 2000, University of York, York, UK, 29-31 March, 2000.

[7] G. Riva, E. Molinari, F. Vincelli, "Interaction and Presence in the Clinical Relationship: Virtual Reality (VR) as a Communicative Medium Between Patient and Therapist”, IEEE Transactions on Information Technology in Biomedicine, 2002, Vol. 6, pp. 198-205.

[8] F. Vincelli, "From Imagination to Virtual Reality: The Future of Clinical Psychology”, CyberPsychology \& Behavior, 1999, Vol. 2 pp. 241-248.

[9] F. Vincelli, G. Riva, "Virtual Reality: A New Tool For Panic Disorder Therapy”, Expert Review of Neurotherapeutics, 2002, Vol. 2 pp. 377383.

[10] G. Gonzalez, "Bilingual Telephone-Assisted Computerized Speech Recognition Interviewing: Automated Screening of Depression Symptoms”, Computers in Psychology Conference 2000, University of York, York, UK, 29-31 March, 2000.

[11] T. Richter, J. Naumann, "Computer-Based Assessment of Reading Skills”, Computers in Psychology Conference 2000, University of York, York, UK, 29-31 March, 2000.

[12] A.W. MacRae, "Multimedia Statistics Course on CD: The First Year's Experience”, Computers in Psychology Conference 2000, University of York, York, UK, 29-31 March, 2000.
[13] E. J. Morris, E. Scanlon, R. Joiner, "Evaluating Multimedia Resources For Teaching Statistics to Psychology Undergraduates", Computers in Psychology Conference 2000, University of York, York, UK, 29-31 March, 2000.

[14] L.G. Gommella, “The Wild, Wild Web”, Semin Urologic Oncol, 2000, Vol. 18, No. 3, pp. 167-171.

[15] G. Eysenbach, J. Powell, O. Kuss, "Empirical Studies Assessing the Quality of Health Information for Consumers on the World Wide Web: A Systematic Review”, JAMA, 2002, 287:2691-2700.

[16] S.C. Matthews, A. Camacho, P.G. Mills, et al, "The Internet for Medical Information About Cancer: Help or Hindrance?”, Psychosomatics, 2003, 44:100-103.

[17] J. Fogel, S.M. Albert, F. Schnabel, "Quality of Health Information on the Internet”, JAMA, 2001, 286:2093-2095.

[18] J. Fogel, "Internet Breast Health Information: Use and Coping Among Women with Breast Cancer”, CyberPsychology \& Behavior, 2004, Vol. 7, No. 1.

[19] N. R. Carlson, W. Buskist, Psychology: The Science of Behavior, Allyn and Bacon, Inc., 1997

[20] Operational Programme for Education and Initial Vocational Training, http://www.epeaek.gr/epeaek/en/home.html

Athanasios S. Drigas (M'86). This author became a Member (M) of IEEE in 1986 (Communications, Education and Computer Societies). He was born in Thessaloniki, Greece in 1959. In 1983 he earned his M.Sc. degree in Electrical Engineering form the University of Patras in Greece. Between 1985 and 1989 he undertook his $\mathrm{PhD}$ thesis in the "Management of computer and broad band networks" at the Informatics Sector of the Technical University of Athens, Greece. Finally, in 2000 he earned his bachelor degree in Psychology from the University of Athens, Greece.

He was the Operational Manager of the Academic Network of Greece (Ariadne) for 14 years (1985-1989). He is the Founder and Scientific Coordinator of Net Media Lab of the National Scientific Research Centre 'Demokritos' in Athens, Greece since 1996 and has been the Scientific Coordinator of more than 25 National and International Projects. His current research interests and activities span the area of e-services (e-learning, ecommerce, e-culture, e-government, e-psychology etc)

Dr. Drigas is the author of more than 70 scientific articles (published in International Journals and Conferences) and of more that 110 National articles. Finally, Dr. Drigas is the author of 7 books on Networks and Services and editor of more than 25 educational CD-ROMs. 